\title{
Desempeño productivo y económico de toretes (Bos taurus) alimentados con concentrados con base en insumos locales
}

\section{Productive and economic performance of bulls (Bos taurus) fed with local based by- products}

\author{
Nilber Gordillo-Vásquez ${ }^{1, a, *}$, Segundo José Zamora-Huamán²,b, Bety P. Canta-Ventura ${ }^{1, c,}$ \\ Wilmer Bernal ${ }^{3, d}$, Flor L. Mejía ${ }^{2, e}$
}

\footnotetext{
${ }^{1}$ Universidad Nacional Toribio Rodríguez de Mendoza de Amazonas, Chachapoyas, Perú.

${ }^{2}$ Facultad de Ingenieria Zootecnista, Agronegocios y Biotecnología, Universidad Nacional Toribio Rodríguez de Mendoza de Amazonas, Chachapoyas, Perú.

${ }^{3}$ USAID- Genex CDP Perú (United States Agency for International Development), Huanuco, Perú.

a Bach., gordillo.0690@ @otmail.com, (1D https://orcid.org/0000-0002-2100-8875

b M.Sc., $\bigotimes$ jose.zamora@untrm.edu.pe, (D) https://orcid.org/0000-0003-3362-9609

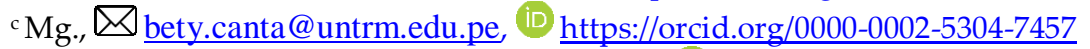

d M.Sc., $\bigotimes_{\text {wilmer.genexcdp.peru@gmail.com, } \mathbb{1 D} \text { https://orcid.org/0000-0003-3482-8466 }}$

eM.Sc., 囚 $\underline{\text { flor.mejia@untrm.edu.pe, (D) https://orcid.org/0000-0002-1851-1285 }}$

* Autor de Correspondencia: Tel. +51 938156583

http://dx.doi.org/10.25127/riagrop.20221.779
}

http://revistas.untrm.edu.pe/index.php/RIAGROP revista.riagrop@untrm.edu.pe

Recepción: 15 de octubre 2021

Aprobación: 02 de diciembre 2021

Este trabajo tiene licencia de Creative Commons. Attribution-NonCommercial-ShareAlike $\quad 4.0$ International Public License - CC-BY-NC-SA 4.0

\section{Resumen}

En el presente trabajo, se evaluó el desempeño productivo y económico de toretes de la raza Brown Swiss. Durante 12 semanas, se utilizaron 15 toretes machos enteros con pesos promedio de $260 \pm 30 \mathrm{~kg}$ y en edades de $12 \pm 2$ meses, divididos en 3 tratamientos: Solamente pastoreados $(\mathrm{P})$; adicionalmente suplementados con alimentos balanceados en base a insumos locales (P+CL) y suplementados con un concentrado comercial $(\mathrm{P}+\mathrm{CC})$. El diseño estadístico fue completamente aleatorizado. Se evaluó la ganancia de peso diaria (GPD) y total (GPT), consumo de materia seca total (CMS) y de forraje (CMS f), conversión alimenticia (CA) y rendimiento en carcasa (RC). De igual manera, fueron evaluados los indicadores económicos (rentabilidad operativa [RO] y la relación beneficio-costo [B/C]). Los valores de GPD (0.99, 0.92 vs 0.75$)$, GPT (83.20, 77.20 vs 62.60$)$, CMS (7.91, 7.76 vs 7.52$)$ y RC (161.30, 157.66 
vs 149.55) fueron estadísticamente superiores $(\mathrm{P}<0.05)$ en los tratamientos $\mathrm{P}+\mathrm{CC}, \mathrm{P}+\mathrm{CL}$ versus $\mathrm{P}$ respectivamente. Los índices económicos, como la $\mathrm{RO}(5.05,5.27$ vs 9.44$)$ y B/C (1.05, 1.06 vs 1.10$)$, fueron más eficientes en el tratamiento sin suplementación $(P)$. Se puede concluir que, en las condiciones de producción evaluadas, el uso de un concentrado local mejoró el rendimiento productivo en toretes y fue más eficiente comparado con su similar comercial. Sin embargo, el grupo que no recibió suplemento o pastoreado, fue más eficiente económicamente.

Palabras claves: desempeño económico. engorde de toretes, indicadores productivos, insumos no tradicionales.

\begin{abstract}
In the present assessment, productive and economic performance in Brown Swiss breed bulls were evaluated. During 12 weeks, 15 whole male bulls with average weights of $260 \pm 30 \mathrm{~kg}$ and $24 \pm 2 \mathrm{~m}$ were used, divided into 3 treatments that were only grazed $(\mathrm{P})$, and supplemented with feeds based on local by products $(\mathrm{P}+\mathrm{CL})$ and a commercial feed $(P+C C)$. The statistical design was completely randomized. Daily weight gain (GPD) and total weight gain (GPT), total dry matter (CMS) and forage (CMS f) consumption, feed conversion (CA) and carcass yield (RC) were evaluated. Similarly, the economic indicators (operating profitability [RO] and benefit-cost ratio [B / C]) were evaluated. The values of GPD (0.99, 0.92 vs 0.75), GPT (83.20, 77.20 vs 62.60), CMS $(7.91,7.76$ vs 7.52) and RC $(161.30,157.66$ vs 149.55$)$ were statistically higher $(\mathrm{P}<0.05)$ in the $\mathrm{P}+\mathrm{CC}, \mathrm{P}+$ CL treatments versus P treatment respectively. Economic indices such as RO (5.05, 5.27 vs 9.44) and B / C (1.05, 1.06 vs 1.10) were more efficient in the treatment without supplementation ( $P$ ). It can be concluded that, under the production conditions evaluated, the use of a local based by products concentrate improved the productive performance in bulls and was more efficient compared to its commercial similar, however, the group that did not receive a supplement or grazed was more economically efficient
\end{abstract}

Keywords: economic performance, bull fattening, productive performance, non traditional feedstuffs.

\section{INTRODUCCIÓN}

El crecimiento de la población a nivel mundial y las limitaciones ambientales continuarán presionando a los productores de carne de vacuno y a las industrias de carne para mejorar la productividad $y$ eficiencia en base al mejoramiento genético, prácticas de manejo nutricional y la sostenibilidad debido a que un manejo adecuado de los sistemas productivos permite el secuestro de carbono y mejora la salud del suelo por el aumento de la materia orgánica (Cusack et al., 2020; Greenwood, 2021; Toro-Mujica, 2021). Por ello, el sector agroalimentario debe cumplir la función de abastecimiento de alimentos seguros que satisfagan el incremento de la demanda mundial de carne de res y la generación de servicios de carácter social y medioambiental (Horrillo et al., 2020; Iraola et al., 2020).

La producción de ganado de carne, conocido como engorde de ganado (EG), no es una actividad especializada como en otros países de Sudamérica (Argentina o Brasil). Esta actividad presenta muchas deficiencias, entre ellas: baja calidad genética (razas especializadas), ausencia de asesoría en manejo y sanidad (Mendoza-Martínez et al., 2008). Además, basa su alimentación en el aprovechamiento de recursos existentes en las tres regiones naturales 
de Perú (MINAGRI, 2001), en pastos naturales y cultivados. Sin embargo, la disponibilidad y calidad de estos se ve influenciada por factores ambientales (lluvias) y se hace necesario suplementar en época de sequía (Godoy et al., 2019). En las zonas de crianza extensiva de sierra y selva de Perú, o en condiciones de sistemas pastoriles intensivos de Uruguay, el EG se fundamenta en un sistema de pastoreo abierto desde las primeras horas de la mañana, que depende de un consumo voluntario y de la digestibilidad, que provoca la rotación de potrero en función a la calidad de forraje, cantidad de animales, así como de las fluctuaciones estacionales (Clariget et al., 2021; Evitayani et al., 2004).

La mayor parte de la ganadería se clasifica como mediana y pequeña, con producciones de subsistencia (PROMPERU, 2017), caracterizadas por baja cantidad y calidad de los pastos que limitan el consumo voluntario y afectan la producción del ganado de carne (Benítez et al., 2007). Por ello, se debe realizar investigaciones enfocadas en identificar especies que sobresalgan por su adaptación al medio, producción de biomasa, persistencia, calidad nutricional y aceptabilidad por parte de los animales (Castro-Rincón et al., 2021). En la constante búsqueda de alternativas que permitan corregir las deficiencias, mejorar las ganancias de peso por unidad de tiempo y con costos apropiados (Delgado et al., 2012), se plantea como alternativa el uso del pastoreo y la suplementación con dietas elaboradas en base a insumos locales como: subproductos de la industria del cultivo del arroz (Oriza sativa), corona de arracacha (Arracacia xanthorrhiza), pulpa de café (Coffea arábica), entre otros. Por ello, la presente investigación tuvo el objetivo de evaluar los índices productivos (consumo de alimento, ganancia de peso, conversión de alimento y rendimientos de carcasa) e índices económicos (utilidad, rentabilidad operativa, y relación beneficio-costo) en toretes alimentados con pastoreo extensivo y suplementados con concentrado elaborados con insumos locales.

\section{MATERIALES Y MÉTODOS}

\subsection{Lugar de estudio}

El experimento se realizó en el anexo El Granero, distrito de El Tingo, provincia de Luya, región Amazonas, ubicado a una altitud de 2893 m s. n. m., con precipitaciones promedio anual de $2031 \pm 71 \mathrm{~mm}$, con una temperatura y humedad promedio diaria de $15 \pm 5^{\circ} \mathrm{C}$ y $90 \pm 12$ $\%$ respectivamente, clima templado-frío (CLIMATE-DATA, 2019).

\subsection{Semovientes y tratamientos}

Se usó un total de 15 toretes enteros de la raza criolla mejorada con raza Brown Swiss en edades de $12 \pm 2 \mathrm{~m}$, con pesos de $260 \pm 30 \mathrm{~kg}$, identificados y desparasitados (Triclabendazol $1 \mathrm{~mL} / 15 \mathrm{~kg}$ de peso vivo [PV] vía oral; Ivermectina $1 \mathrm{~mL} / 50 \mathrm{~kg}$ PV) y vitaminas A, D3 y E (5 mL por cabeza vía intra muscular). El diseño empleado para la investigación fue un Diseño Completo al azar (DCA), de tres tratamientos con 5 repeticiones. Los animales fueron divididos en 3 tratamientos: $\mathrm{T} 1=$ pastoreo + concentrado comercial $(\mathrm{P}+\mathrm{CC}) ; \mathrm{T} 2=$ pastoreo + concentrado local $(\mathrm{P}+\mathrm{CL})$ y $\mathrm{T} 3=$ solo pastoreo $(\mathrm{P})$, con 5 repeticiones (toretes) por tratamiento. Todos los animales fueron pastoreados diariamente de manera rotacional convencional en horarios de $8.30 \mathrm{~h}$ hasta las $16.00 \mathrm{~h}$ y ocuparon un área total aproximada de 3.5 ha por tratamiento, con una carga animal de 
1.5 animales/ha. La dieta base forrajera estuvo conformada por las proporciones de 9:11:1:1.2 respectivamente y suministrada por un periodo de 12 semanas, más dos semanas de adaptación al consumo de las dietas experimentales. La dieta experimental se suministró de manera diaria por las mañanas a razón de $2 \%$ del peso metabólico de los animales ( $\left.\mathrm{PV}^{0.75}\right)$.

\subsection{Elaboración de dietas experimentales y composición nutricional}

Antes del ingreso de los animales a los potreros experimentales, se determinó la composición, bromatológica de la dieta base forrajera por triplicado (Dactylis glomerata [PO, pasto ovillo], Paspalum candidum [PN, pasto nudillo], Philoglosa mimuloides [PS, pasto siso], Trifolium repens [TR, trébol]) (Tabla 1 ). La dieta con insumos locales (CL) fue formulada mediante MS Excel - Solver y para cubrir las necesidades nutritivas de ganado de carne, se basó en recomendaciones de NRC (2000) (Tabla 2 y tabla 3) y en base a lo propuesto por Galoc et al., (2021) y Yoplac et al., (2021). Se realizó el análisis químico proximal por triplicado (materia seca [MS], proteína bruta [PB], extracto etéreo [EE], cenizas [Cza]) en base a AOAC (1995), fibra detergente neutro [FDN; Van Soest, (1994)], energía bruta con la metodología propuesta por Givens et al., (1989) en bomba calorimétrica (Parr 6200 Calorimeter, USA) y digestibilidad in vitro en incubador DaisyII@ (Ankom Technology A200, USA) en base a la metodología propuesta por Giraldo et al. (2007).

\subsection{Indicadores productivos}

Antes de ser alimentados, todos los animales fueron pesados cada semana (08:30) con una cinta bovino métrica. Los valores de consumo de alimento se realizaron de manera diaria, descontando y pesando el sobrante de alimento. La conversión alimenticia se calculó como la relación entre el consumo de alimento $(\mathrm{kg})$ y la ganancia de peso obtenida por semana. Al final del experimento (12 semanas), los animales fueron puestos en ayuno de 12 horas $y$ solamente con ingesta de agua, trasladados al camal en donde fueron sacrificados para evaluar el rendimiento de carcasa: animal sacrificado y eviscerado (sin cabeza, piel, patas ni vísceras).

Tabla 1. Composición química nutricional de las dietas experimentales $(\overline{x \pm D E})$

\begin{tabular}{lcrrrrrr}
\hline Alimento & MS & PB & EE & \multicolumn{1}{c}{ Cza } & FDN & EB & DIVMS \\
\hline Trifolium repens & $22.0 \pm 1.39$ & $18.3 \pm 0.14$ & $1.98 \pm 0.52$ & $9.77 \pm 1.73$ & $50.15 \pm 0.94$ & $4.2 \pm 0.88$ & $81.22 \pm 0.02$ \\
Dactylis glomerata & $18.2 \pm 1.30$ & $8.88 \pm 0.68$ & $2.88 \pm 0.44$ & $7.36 \pm 0.81$ & $54.15 \pm 2.45$ & $4.12 \pm 0.33$ & $77.51 \pm 3.15$ \\
Philoglosa mimuloides & $10.3 \pm 2.08$ & $13.84 \pm 1.60$ & $1.89 \pm 0.33$ & $13.84 \pm 1.50$ & $33.44 \pm 3.40$ & $3.8 \pm 0.28$ & $83.12 \pm 0.25$ \\
Paspalum candidum & $19.2 \pm 0.28$ & $9.84 \pm 1.41$ & $2.55 \pm 0.49$ & $7.41 \pm 1.58$ & $54.25 \pm 1.91$ & $4.12 \pm 0.11$ & $69.3 \pm 0.39$ \\
\hline
\end{tabular}


Tabla 2. Composición del concentrado local

\begin{tabular}{lr}
\hline \multicolumn{1}{c}{ Alimento } & \multicolumn{1}{c}{$\%$} \\
\hline Maíz amarillo & 22.77 \\
Torta de soya & 7.00 \\
Melilotus albus & 13.00 \\
Arracacia $x a n t h o r r i z a^{\prime}$ & 3.00 \\
Pulpa de café & 15.00 \\
Arroz quebrado & 11.00 \\
Polvillo de arroz & 18.00 \\
Melaza & 8.00 \\
Urea $^{\text {Aditivos }}{ }^{1}$ & 0.48 \\
Total & 0.18 \\
Costo (S/ x kg) & $\mathbf{1 0 0 . 0}$ \\
\hline${ }^{1}$ Aditivos: Mezcla de sales minerales, calcio
\end{tabular}

Tabla 3. Valor nutricional $(\overline{x \pm D E})$ del concentrado local

\begin{tabular}{lrr}
\hline \multicolumn{1}{c}{ Componente } & \multicolumn{1}{c}{ CL $^{1}$} & \multicolumn{1}{c}{ CC $^{2}$} \\
\hline Materia seca, \% & $90.16 \pm 1.12$ & $92.33 \pm 1.40$ \\
Proteína bruta, \% & $14.00 \pm 0.08$ & $14.50 \pm 0.19$ \\
Extracto etéreo, \% & $5.20 \pm 0.61$ & $5.33 \pm 0.89$ \\
$\mathrm{FDN}^{3}, \%$ & $19.57 \pm 0.36$ & $20.33 \pm 0.44$ \\
$\mathrm{ENm}^{4}, \mathrm{Mcal} / \mathrm{kg}$ & $1.63 \pm 0.08$ & $1.77 \pm 0.12$ \\
$\mathrm{ENg}^{5}, \mathrm{Mcal} / \mathrm{kg}$ & $1.12 \pm 0.21$ & $1.18 \pm 0.15$ \\
\hline
\end{tabular}

${ }^{1} \mathrm{CL}$ : concentrado local. ${ }^{2} \mathrm{CC}$ : concentrado comercial. ${ }^{3} \mathrm{FDN}$ : Fibra detergente neutro. ${ }^{4} \mathrm{ENm}$ : Energía neta calculada en base a Garrett (1980). ${ }^{5} \mathrm{ENg}$ : Energía neta calculada en base a Boschini-Figueroa (2006).

\subsection{Indicadores de rentabilidad}

Se evaluó el índice de rentabilidad operativa, medido como la relación entre la utilidad operativa y las ventas netas (costo de inversión) (Weinberger, 2009). Además, se determinó la relación beneficio-costo $(\mathrm{B} / \mathrm{C})$ que compara la relación entre el valor actual de los ingresos con el valor actual de los egresos. Si la relación B/C es mayor o igual a 1 , el proyecto debe ser aceptado, si el valor B/C es menor a 1 debe ser rechazado (Weinberger, 2009).

\subsection{Análisis estadístico}

Los índices evaluados productivos fueron analizados en el marco de un diseño completamente al azar y con el modelo lineal general, con análisis de varianza (ANOVA) de una vía (tipo de alimento como factor fijo) para determinar si las medias de dos o más grupos son diferentes. De existir diferencias significativas $(\mathrm{P}<0.05)$, se usó la prueba posterior de rango de Tukey. Los datos fueron evaluados usando el paquete informático SPSS, versión 25.

\section{RESULTADOS Y DISCUSIÓN}

\subsection{Indicadores productivos}

Al final del experimento, los valores promedio de pesos finales, ganancia promedio diaria de peso y ganancia promedio total de peso en los tratamientos $\mathrm{P}+\mathrm{CC}$ y $\mathrm{P}+\mathrm{CL}$ fueron superiores de manera significativa $(\mathrm{P}<0.05)$ comparados con el tratamiento $\mathrm{P}$. El consumo de materia seca diaria en el tratamiento $\mathrm{P}+\mathrm{CC}, \mathrm{P}+\mathrm{CL}$ fue superior de manera significativa $(\mathrm{P}<0.05)$ y un mayor consumo de materia seca en forraje se pudo evidenciar en el tratamiento P. Sin embargo, no hubo diferencias en los valores para conversión alimenticia. Al final del experimento mayores rendimientos de carcasa se encontraron en los tratamientos $\mathrm{P}+\mathrm{CC}$ y $\mathrm{P}+\mathrm{CL}$ comparado con el tratamiento $\mathrm{P}$ (semana 12) de consumo de materia seca (tabla 4). 
Tabla 4. Comportamiento productivo en toretes de engorde

\begin{tabular}{lrrrrr}
\hline \multicolumn{1}{c}{ Concepto } & \multicolumn{1}{c}{ P+CC } & \multicolumn{1}{c}{ P+CL } & \multicolumn{1}{c}{ P } & EEM & P valor \\
\hline Peso inicial $(\mathrm{kg})$ & 227.00 & 226.00 & 225.00 & 2.61 & 0.959 \\
Peso final $(\mathrm{kg})$ & $310.20^{\mathrm{a}}$ & $303.20^{\mathrm{a}}$ & $287.60^{\mathrm{b}}$ & 3.27 & 0.004 \\
GPD (kg) & $0.99^{\mathrm{a}}$ & $0.92^{\mathrm{a}}$ & $0.75^{\mathrm{b}}$ & 0.03 & 0.001 \\
GPT (kg) & $83.20^{\mathrm{a}}$ & $77.20^{\mathrm{a}}$ & $62.60^{\mathrm{b}}$ & 2.80 & 0.001 \\
CMS (kg/día) & $7.91^{\mathrm{a}}$ & $7.76^{\mathrm{ab}}$ & $7.52^{\mathrm{b}}$ & 0.04 & 0.001 \\
CMS f (kg/día) & $5.93^{\mathrm{b}}$ & $5.82^{\mathrm{b}}$ & $7.52^{\mathrm{a}}$ & 0.07 & $<0.001$ \\
CA & 9.55 & 10.10 & $10.38^{\mathrm{a}}$ & 0.25 & 0.120 \\
RC (kg) & $161.30^{\mathrm{a}}$ & $157.66^{\mathrm{a}}$ & $149.55^{\mathrm{b}}$ & 1.70 & 0.004 \\
\hline
\end{tabular}

GPD: ganancia peso promedio diario; GPT: Ganancia de peso total; RC: Rendimiento de carcasa; CMS: Consumo de materia seca; CMSf: Consumo materia seca de forraje; CA: conversión alimenticia; RC: rendimiento carcasa.

Los factores que afectan el control de consumo de alimentos son complejos y multifactoriales (Forbes 2007). En el presente experimento, la mezcla de forrajes, con un bajo contenido en materia seca (suculentos), y el uso de concentrados permitieron un mayor CMS total en los tratamientos $\mathrm{P}+\mathrm{CC}(7.91 \mathrm{~kg} / \mathrm{d})$ y $\mathrm{P}+\mathrm{CL}$ $(7.76 \mathrm{~kg} / \mathrm{d})$, comparado con $7.77 \mathrm{~kg} / \mathrm{d}$ (Rodrigues et al., 2013). De igual manera, la restricción de concentrados en el tratamiento $\mathrm{P}$ determinó un mayor CMS f $(7.52 \mathrm{~kg} / \mathrm{d})$, comparado con $9.87 \mathrm{~kg}$ MS/día y $7.76 \mathrm{Kg}$ CMS f/d (Aharoni et al., 2004).

Los trabajos realizados por Clímaco et al. (2011) en bovinos de diferente genética cebú (Tabapua y Bonsmara) y sus cruces con Angus encontraron valores superiores en ganancia de peso diario (0.93 kg y $1.12 \mathrm{~kg} /$ día), semejantes al trabajo de Mamani (2021), quien reportó ganancias de 1.64 y 1.21 kg/día en una alimentación mixta en toretes del biotipo Aberdeen Angus y Criollo en ecosistemas de altura. Del mismo modo, estos resultados fueron superiores al reporte de Graillet-Juarez et al. (2017), quienes obtuvieron incremento de peso de $0.494 \mathrm{~kg} /$ día al suplementarse con bloques nutricionales. También fue superior a los resultados obtenidos por Quiceno et al. (2012), quienes evaluaron el incremento de peso de toretes Blanco Orejinegro y Romosinuano, donde el incremento de peso fue de 0.50 y 0.37 kg/animal/día, respectivamente.

Los resultados obtenidos en este estudio fueron mayores a la conversión alimenticia obtenida por Vaz et al. (2013), quienes reportan conversiones alimenticias de 4.78 a 5.51 en ganado vacuno criados bajo un sistema de feedlot. Al evaluar la conversión alimenticia en terneros machos criados en feedlot y sistema silvopastoril, Fernandes et al. (2004) reportó valores de 7.1 y 9.8, respectivamente. Estos valores fueron menores a los encontrados en este estudio. Al respecto, Crews (2005) y Herd et al. (2003) mencionaron que estas diferencias están relacionadas con la genética e indican que los animales mejorados presentan mejores conversiones superiores respecto a los criollos. El uso de concentrados en bovinos al pastoreo permite aumentar la cantidad de energía, porque es aprovechada por los microrganismos en el rumen (Pordomingo, 2003). El engorde 
rápido típicamente de un novillo joven, destinado al sacrificio y confinado hasta su peso óptimo de mercado, y si se consideran los sistemas extensivos o semintensivos, se define como sistema de producción que hace hincapié en el crecimiento y desarrollo de los animales sobre la deposición de grasa y buena conversión alimenticia (Callejas-Juárez, 2017). El uso de concentrado facilita la degradación de insumos por los microrganismos ruminales y la absorción de nutrientes por parte del organismo animal (Herd et al., 2003). Esto indicaría que tanto el concentrado comercial (CC), como el uso de insumos en la preparación de un concentrado local (CL), mejoran el CMS y podrían sustituir a los insumos tradicionales como torta de soya, afrecho de trigo, entre otros.

\subsection{Indicadores económicos y de rentabilidad} Se encontró mayores costos de producción en el tratamiento $\mathrm{P}+\mathrm{CC}$, seguido por los tratamientos $\mathrm{P}+\mathrm{CL}$ y PAST. Los costos relacionados con animales, sanidad y mano de obra se sumaron en un solo grupo por ser montos fijos (S/ 8800.00) y los valores de mano de obra se han calculado en base a 28 jornales por tratamiento (S/ 25.00). El costo de forraje se valorizó en base al costo de alquiler de terreno por hectárea (S/0.10 por kg) (figura 1).
Una mayor utilidad se encontró en el tratamiento $\mathrm{P}$ con $\mathrm{S} / 950.16$ en comparación con el tratamiento P+CC (S/ 548.44) y P+CL (S/ 559.56), con mayores valores de rentabilidad operativa para el tratamiento a base de pastoreo (9.44). Además, se evidenció mayor relación beneficio/costo $(\mathrm{B} / \mathrm{C})$ en el tratamiento de $\mathrm{P}$ (1.10), cuya interpretación está dado por una ganancia de $\mathrm{S} / 0.10$ por cada sol invertido (tabla $5)$.

Se pudo evidenciar la factibilidad de un modelo de negocio de producción de carne bovina como se realiza en otros países, como Chile, Reino Unido, Irlanda y Nueva Zelandia (Werner y López, 2021). Los resultados muestran la necesidad que tienen las explotaciones ganaderas de aumentar sus ingresos. En el presente trabajo, los toretes engordados con la suplementación de concentrados $(\mathrm{P}+\mathrm{CC} \mathrm{y}$ $\mathrm{P}+\mathrm{CL}$ ) lograron mayores ingresos económicos, pero también se necesitó de mayores costos de producción, ocasionado por la compra de insumos para el alimento balanceado (Morao y Rüegger, 2011; Nuñez, 2019). Con estos valores, se pudo evidenciar mayor utilidad operativa, mayor rentabilidad y finalmente una mejor relación beneficio/costo $(\mathrm{B} / \mathrm{C})$ en el tratamiento P.

Tabla 5. Indicadores económicos y de rentabilidad en toretes de engorde

\begin{tabular}{lrrr}
\hline \multicolumn{1}{c}{ Concepto } & P+CC & \multicolumn{1}{c}{ P+CL } & \multicolumn{1}{c}{ P } \\
\hline (+) $\operatorname{Ventas}^{1}(\mathrm{~S} /)$ & 10857.54 & 10612.00 & 10066.00 \\
(-) Costos & 10309.10 & 10052.44 & 9115.84 \\
(=) Utilidad operativa & 548.44 & 559.56 & 950.16 \\
Rentabilidad Operativa & \\
Relación Beneficio-Costo $^{3}$ & 5.05 & 5.27 & 9.44 \\
\hline
\end{tabular}

${ }^{1}$ Ventas: Total kg en 5 animales x 7 (S/ kg venta en pie) ${ }^{2}$ Rentabilidad operativa: Utilidad operativa/ventas ${ }^{3}$ Relación beneficio-costo: Valor Actual Ingresos/Valor actual egresos 


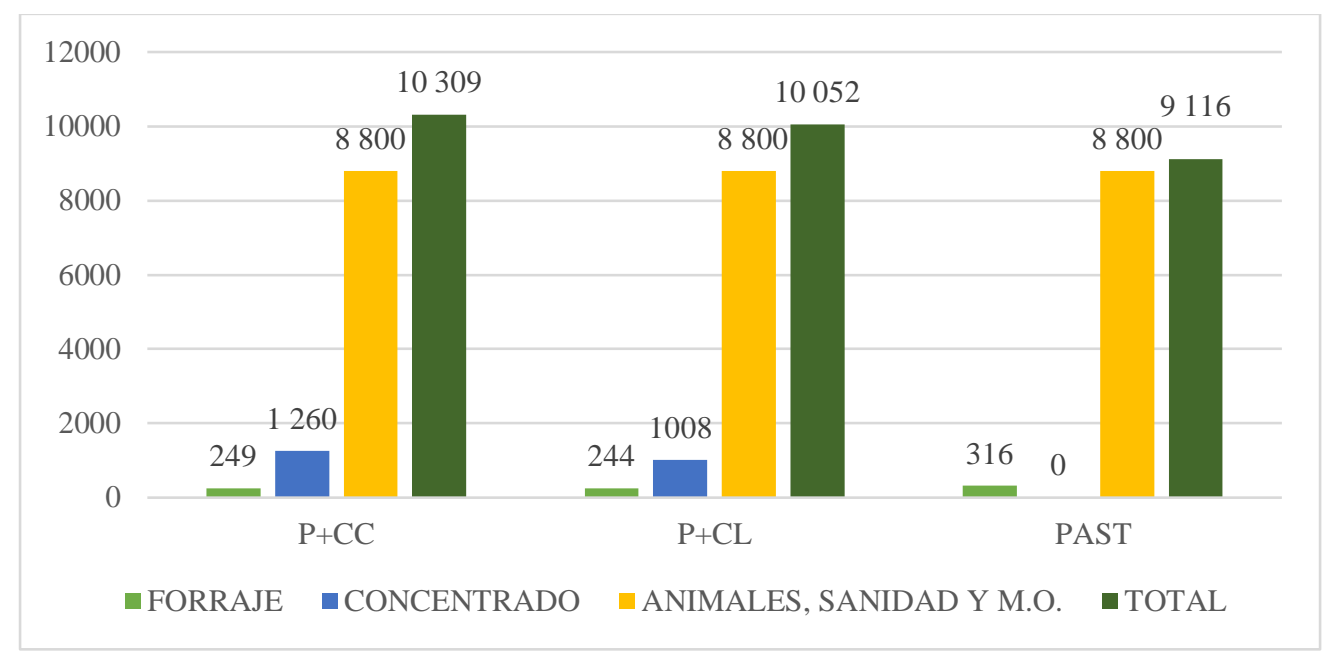

Figura 1. Costos en proceso de engorde de toretes (S/).

\section{CONCLUSIONES}

En las condiciones de producción evaluadas, el uso de un concentrado local mejoró el rendimiento productivo en toretes, porque logró una mayor eficiencia en comparación del uso de un concentrado comercial. Sin embargo, el grupo que no recibió ningún tipo de concentrado fue más eficiente, económicamente.

\section{Declaración de intereses}

Ninguna.

\section{Agradecimientos}

Los autores agradecen al proyecto 189-2015FONDECYT-DE

“Caracterización bromatológica de insumos no tradicionales para la alimentación animal de la región Amazonas", por financiar la ejecución del presente estudio.

\section{Referencias}

Aharoni, Y., Brosh, A., Orlov, A., Shargal, E. y Gutman, M. (2004). Measurements of energy balance of grazing beef cows on Mediterranean pasture, the effects of stocking rate and season. Livestock Production Science, 90(2-3),

89-100.
Https://doi.org/10.1016/j.livprodsci.2004.03.007

AOAC. (1995). Official methods of analysis of AOAC international. Association of Official Analytical Chemists.

Benítez, D., Ramírez, A., Díaz, M., Ray, J., Guerra, J., \& Vegas, A. (2007). Comportamiento de machos vacunos en un sistema racional de pastoreo en el Valle del Cauto. Revista Cubana de Ciencia Agrícola, 41(3), 227-230.

Boschini-Figueroa, C. (2006). Proteicas de la morera (Morus alba) aprovechables. Agronomía mesoamericana, 10.

Callejas-Juárez, N., Rebollar-Rebollar, S., OrtegaGutierrez, J.A. \& Domínguez-Viveros, J. (2017). Parámetros bio-económicos de la producción intensiva de la carne de bovino en México. Rev Mex Cienc Pecu, 8(2), 129-138. DOI: https://doi.org/10.22319/rmcp.v8i2.4415

Castro-Rincón, E., Carulla-Fornaguera, J.E. \& CárdenasRocha, E.A. (2021). Calidad nutricional de cinco gramíneas asociadas a Lotus uliginosus Schkuhr en el trópico alto de Colombia. Pastos y Forrajes, 44.

Clariget, J.M., Lema, O.M., La Manna, A., Perez, E., Banchero, G. \& Fernández, E. (2021). Estimated beef cattle performance under intensive grazing systems in Uruguay. Agrociencia Uruguay, 25(1),e107. Https://doi.org/10.31285/AGRO.25.107

Clímaco, S.M., Ribeiro, E.L., Mizubuti, Y., Silva, L., Das, D.F., Barbosa, M.A. \& Bridi, A.M. (2011). Desempenho e características de carcaça de bovinos de corte de quatro grupos genéticos terminados em confinamento. Revista Brasileira de Zootecnia, 40(7), 1562-1567. Https://doi.org/10.1590/S151635982011000700023

CLIMATE-DATA. (2018). Clima Nuevo Tingo: Temperatura, Climograma y Tabla climática para Nuevo Tingo- 
Climate-Data.org. Https://es.climatedata.org/america-del-sur/peru/amazonas/nuevotingo-438681/

Crews, D.H.D. (2005). Genetics of efficient feed utilization and national cattle evaluation: A review. Genetics and Molecular Research, 4(2), 152-165.

Cusack, D.F., Kazanski, C.E., Hedgpeth, A., Chow, K., Cordeiro, A.L., Karpman, J. \& Ryals, R. (2021). Reducing climate impacts of beef production: A synthesis of life cycle assessments across management systems and global regions. Global Change Biology, 27, 1721-1736. Https://doi.org/10.1111/gcb.15509

Delgado, C.A., Trigueros, V.A., Tang, P. J., Angelats, M.R., \& Gavidia, C.C. (2012). Efecto de un modificador orgánico en la ganancia de peso en ganado cebú en el trópico peruano. Revista de Investigaciones Veterinarias del Perú, 23(2), 153-159. Https://doi.org/10.15381/rivep.v23i2.895

Evitayani, L., Fariani, A., Ichinohe, T. \& Fujihara, T. (2004). Study on Nutritive Value of Tropical Forages in North Sumatra, Indonesia. Asian-Australasian Journal of Animal Sciences, 17(11), 1518-1523. Https://doi.org/10.5713/ajas.2004.1518

Fernandes, H.J., Paulino, M.F., Martins, R.G.R., Valadares, S., Torres, R., Paiva, L. M. \& Moraes, G.F. (2004). Ganho de peso, conversão alimentar, ingestão diária de nutrientes e digestibilidade de garrotes nãocastrados de três grupos genéticos em recria e terminação. Revista Brasileira de Zootecnia, 33(6 suppl 3), 2403-2411. Https://doi.org/10.1590/S151635982004000900029

Forbes, M. (2007). A personal view of how ruminant animals control their intake and choice of food: Minimal total discomfort. Nutrition Research Reviews, 20(2), 132-146. Https://doi.org/10.1017/S0954422407797834

Galoc, N., Vásquez, H.V. \& Bernal, W. (2021). Caracterización nutricional de trece variedades de pastos naturalizados de la región Amazonas. Revista Científica UNTRM: Ciencias Naturales e Ingeniería, 2(3), 29. Https://doi.org/10.25127/ucni.v2i3.601

Garrett, W.N. (1980). Energy utilization by growing cattle as determined in 72 comparative slaughter experiments. En Energy Metabolism (pp. 3-7). Elsevier. Https://doi.org/10.1016/B978-0-408-10641-2.50006-9

Giraldo, L.A., Gutiérrez, L.A. \& Rúa, C. (2007). Comparación de dos técnicas in vitro e in situ para estimar la digestibilidad verdadera en varios forrajes tropicales. Revista Colombiana de Ciencias Pecuarias, 20(3), 269-279.

Givens, D.I., Everington, J.M. \& Adamson, A.H. (1989).
The digestibility and metabolisable energy content of grass silage and their prediction from laboratory measurements. Animal Feed Science and Technology, 24(1-2), 27-43. Https://doi.org/10.1016/03778401(89)90018-7

Godoy, D., Fernandez, M., Gomez, C. \& Hidalgo, V. (2019). Suplementación nutricional de vacunos en el trópico Peruano. Manual, Universidad Nacional Agraria La Molina. Recuperado de https://www.researchgate.net/publication/335541947 _Suplementacion_nutricional_estrategica_para_gan ado_vacuno_en_el_tropico_peruano

Graillet-Juarez, E.M., Arieta-Román, R.J., Aguilar-Garza, M.C., Alvarado-Goméz, L.C. \& Orozco, N.R. (2017). Ganancia de peso diario en toretes de iniciación en pastoreo suplementados con bloques nutricionales. REDVET. Revista Electrónica de Veterinaria, 18(1). Https://www.redalyc.org/articulo.oa?Id=6364968401 0

Greenwood, P. (2021). An overview of beef production from pasture and feedlot globally, as demand for beef and the need for sustainable practices increase. Animal. The international journal of animal biosciences. Science Direct. ELSEVIER. Australia. Http://doi.org/10.1016/j.animal-2021.100295

Herd, R.M., Archer, J.A. \& Arthur, P.F. (2003). Reducing the cost of beef production through genetic improvement in residual feed intake: Opportunity and challenges to application1. Journal of Animal Science, 81(13_suppl_1), E9-E17. Https://doi.org/10.2527/2003.8113_suppl_1e9x

Horrillo, G., Gaspar, G., Diaz, C. \& Escribano, S. (2020). Análisis económico-estructural de explotaciones ganaderas ecológicas en dehesas y pastizales de Extremadura. Revista Española de Estudios Agrosociales y pesqueros, 256, 133-171 Recuperado el 10 de agosto de

2021 miteco.gob.es/app/publicaciones/art_datos.asp?Arti culoid $=1496 \&$ codrevista $=$ REEAP

Iraola, J., Dios-Palomares, R. \& Barroz-Rodríguez, M. (2020). Propuesta metodológica para el cálculo de indicadores ambientales en el engorde de machos vacunos en pastoreo Revista de Producción Animal 32(2). Recuperado de https://revistas.reduc.edu.cu/index.php/rpa/article/v iew/e3381

Mamani, A. (2021). Efecto de alimentación mixta sobre la ganancia de peso en vacunos del biotipo Aberdeen Angus y Criollo en la etapa de crecimiento en altura. Tesis de Médico Veterinario y Zootecnista. Universidad Nacional del Altiplano de Puno.

Mendoza-Martínez, G., Plata-Pérez, F., EspinosaCervantes, R. \& Lara-Bueno, A. (2008). Manejo 
nutricional para mejorar la eficiencia de utilización de la energía en bovinos. Universidad y ciencia, 24(1), 75-87.

MINAGRI. (2001). Vacunos de doble propósito. Https://www.minagri.gob.pe/portal/40-sectoragrario/situacion-de-las-actividades-de-crianza-yproducci/304-vacunos-de-doble-proposito

Morao, G.A. \& Rüegger, M.J.A. (2011). Desempeño productivo, tipificación y rendimiento a la faena de machos enteros jovenes Holando Argentino engordados a corral. Invet, 13(2), 9-16.

NRC. (2000) Download: Nutrient Requirements of Beef Cattle: Seventh Revised Edition: Update 2000. The National Academies Press. Https://www.nap.edu/download/9791

Nuñez, N. (2019). Comportamiento productivo de toretes criollos para carne con la aplicación de un anabólico (Zeranol) e inmunocastración (análogo de gnrh) en el distrito de Huicungo, provincia de Mariscal Cáceres. Tesis de Médico Veterinario. Universidad Nacional de San Martín- Tarapoto.

Pordomingo, A.J. (2003). Suplementación con granos a bovinos en pastoreo. Sitio de producción animal, 4 .

PROMPERU. (2017). Ganadería en el Perú: El importante desarrollo ganadero en el Perú. Https://peru.info/espe/inversiones/noticias/5/23/el-importantedesarrollo-ganadero-en-el-peru

Quiceno, J., Martinez, R., Mateus, H., Gallego, J. \& Medina, P. (2012). Crecimiento en pastoreo rotacional de toretes de razas criollas Romosinuano y Blanco Orejinegro en Colombia I Revista MVZ Córdoba, 17(1), 2891-2899.

Https://revistamvz.unicordoba.edu.co/article/view/2 58

Rodrigues, É., Arrigoni, M.D., Andrade, C.R., Martins,
C.L., Millen, D.D., Parra, F.S., Jorge, A.M. \& Andrighetto, C. (2013). Performance, carcass characteristics and gain cost of feedlot cattle fed a high level of concentrate and different feed additives. Revista Brasileira de Zootecnia, 42(1), 61-69. Https://doi.org/10.1590/S1516-35982013000100009

SPSS. (2017). Statistical Package for the Social Sciences (25.0) [Computer software]. IBM SPSS Inc.

Toro-Mujica, P. \& González-Ronquillo, M. (2021). Feeding and Nutritional Strategies to Reduce Livestock Greenhouse Gas Emissions Editorial: Frontiers. Vet. Sci., 8, 717426. Doi: 10.3389/fvets.2021.717426

Van Soest, P.J. (1994). Nutritional Ecology of the Ruminant by Peter J. Van Soest. Hardcover. Cornell University Press.

Https://www.cornellpress.cornell.edu/book/9780801 427725/nutritional-ecology-of-the-ruminant/

Vaz, F.N., Restle, J., Flores, J.L., Vaz, R.Z. \& Pacheco, P.S. (2013). Desempenho em confinamento de machos bovinos superjovens de diferentes grupos genéticos. Revista Ciência Agronômica, 44 (1), 167-173. Https://doi.org/10.1590/S1806-66902013000100021

Weinberger, K. (2009). Plan de Negocios: Herramienta para evaluar la viabilidad de un negocio.

Werner, A. \& López, C. (2019). Modelo de negocio para producción de carne bovina en Chile. Universidad de los Lagos, Osorno, Chile.I Revista Académica E Negocios, 4(1), 61- 70.

Yoplac, I.J., Goñas, K., Bernal, W., Vásquez, H.V. \& Maicelo, J.L. (2021). Caracterización química y digestibilidad in vitro de semillas y subproductos agroindustriales amazónicos con potencial para alimentación animal. Revista de Investigaciones Veterinarias del Perú, 32(3), e18765. Https://doi.org/10.15381/rivep.v32i3.18765 